Kurz notiert

Impfempfehlung gegen Masern - Seit einigen Jahren sieht man bei Masern eine Altersverschiebung ins Erwachsenenalter, wo es häufig zu Komplikationen kommt. Deshalb hat die STIKO seit Juli 2010 die Impfempfehlung gegen Masern ausgeweitet: Alle nach 1970 geborenen Personen, die 18 Jahre und älter sind, die bisher nicht geimpft wurden, deren Impfstatus unklar ist oder die nur einmal in der Kindheit geimpft wurden, sollen eine Masern-Impfung erhalten. Diese sollte vorzugsweise mit einem Kombinationsimpfstoff gegen Masern, Mumps und Röteln (MMR) wie z. B. M-M-RvaxPro ${ }^{\oplus}$ erfolgen.

Sanofi Pasteur MSD

ADHS bei Erwachsenen - Zur Behandlung der Aufmerksamkeitsdefizit-/Hyperaktivitätsstörung (ADHS) bei Erwachsenen ist nun Medikinet ${ }^{\circledR}$ adult (Wirkstoff Methylphenidat) zugelassen und wird voraussichtlich im Sommer 2011 erhältlich sein. Damit wird eine Versorgungslücke geschlossen. Bei mehr als der Hälfte der Kinder und Jugendlichen mit ADHS persistiert die Erkrankung bis ins Erwachsenenalter. Dies entspricht rund 250000 erwachsenen ADHS-Patienten in Deutschland. Medice

Nicht kleinzelliges Bronchialkarzinom $>$ In Europa beginnt eine große, multinationale Phase-III-Studie mit Tivantinib (ARQ 197), einem Inhibitor der c-MET-Rezeptor-Tyrosinkinase, zur Therapie des fortgeschrittenen, nicht plattenepithelialen, nicht kleinzelligen Bronchialkarzinom (NSCLC). Tivantinib plus Erlotinib wird mit Placebo plus Erlotinib verglichen bei Patienten, die wegen fortgeschrittenem NSCLC bereits behandelt wurden. Prof. Giorgio Scagliotti, Turin (Italien), Hauptprüfer der Studie für Europa, hofft, dass sich Tivantinib als effektive Therapiemöglichkeit für Patienten mit dieser Erkrankung herausstellen wird.

Daiichi-Sankyo

Opioide bei chronischen Schmerzen

\title{
Weniger Schlafprobleme bei Einmalgabe
}

— Trotz Basisanalgesie leidet die Mehrheit chronischer Schmerzpatienten unter Schlafproblemen und daraus resultierender Tagesmüdigkeit. Dass dies auch von der Applikationsform und dem Dosierschema abhängt, zeigten die nationalen Ergebnisse der Patienten-Präferenz-Umfrage PARES (PAin RESearch). An ihr nahmen 875 Patienten teil, die an mäßigen bis starken chronischen Nicht-Tumor- oder Tumorschmerzen litten und ein WHO-Stufe-II- oder WHOStufe-III-Opioid erhielten. Verglichen wurden die ein-, zwei-, dreimal tägliche und öfter (oral) Applikation sowie die Applikation alle drei oder mehr Tage (transdermal).

$73 \%$ der Befragten berichteten von schmerzbedingten Einschlaf- und $80 \%$ von Durchschlafstörungen. $73 \%$ fühlten sich am Tag nicht ausreichend oder nur einigermaßen erholt und ausgeschlafen. Ein- bzw. Durchschlafstörungen wurden signifikant häufiger bei Dreimalgabe (86\% bzw. 91\%) als bei Einmalgabe (68\% bzw. $73 \%$ ) geschildert.
„Patienten, die dreimal täglich ein Analgetikum einnahmen, waren generell unzufriedener mit ihrer Therapie. Am besten schnitt insgesamt die Einmalgabe ab", so Dr. Gerhard Müller-Schwefe, Göppingen. Die einmal tägliche Gabe ist z. B. mit dem OROS $^{\circledR}$-Hydromorphon Jurnista ${ }^{\circledR}$ möglich. Es zeichnet sich durch eine starke schmerzlindernde und verlässlich Wirkung über das Applikationsintervall aus.

- Red.

Quelle: 22. Deutscher interdisziplinärer Schmerz- und Palliativkongress, Frankfurt am Main (Veranstalter: Janssen-Cilag)

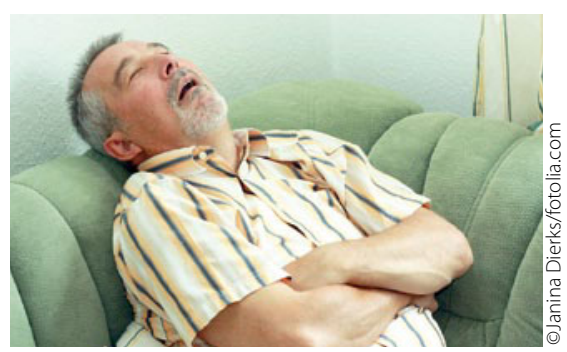

Fast drei Viertel der Schmerzpatienten fühlen sich nicht richtig ausgeschlafen.

\section{Weißdornextrakt bei leichter Herzinsuffizienz Mögliche Wirkung im Endothel-Bereich}

- Weißdornextrakt wird bei leichten Formen der Herzinsuffizienz zur Steigerung der körperlichen Belastbarkeit eingesetzt. Auf Nanotechnik beruhende Forschungsergebnisse zeigen einen möglichen Wirkmechanismus im Bereich des Endothels.

Bekannt ist, dass ein hoher Salzkonsum das Risiko für Hypertonie und für kardiovaskuläre Ereignisse erhöht, so Prof. Dr. Hans Oberleithner, Münster. Dank der Atomic-Force-Mikroskopie (AFM), die eine Darstellung im Nanobereich ermöglicht, konnte nun die mechanische Verformbarkeit von lebenden Endothelzellen je nach Salzkonzentration dargestellt werden. Bereits geringgradige Erhöhungen der Natriumkonzentration führen in wenigen $\mathrm{Mi}$ nuten zu einer Versteifung der Endothelzellen. Diese können nur noch wenig NO produzieren, was zu einer Erhöhung des arteriellen Blutdrucks führt. Auf der Ober- fläche der Endothelzellen befindet sich eine nur wenige Nanometer dicke Pufferzone aus zuckerähnlichen Molekülen (sog. Glykokalyx), die eine schützende Barriere zwischen Blut und Endotheloberfläche darstellt.

Für Weißdornextrakt (Crataegutt $\left.{ }^{\circledR}\right)$ ist eine Steigerung der NO-Produktion im Endothel gezeigt worden. Die endotheliale Glykokalyx könnte hier eine wichtige Rolle spielen. In Konzentrationen, die auch im menschlichen Plasma bei regelmäßiger Einnahme des Phytotherapeutikums erreicht werden, wurde eine Zunahme der Pufferschichthöhe um 22\% und eine Abnahme der Steifigkeit um ca. 30\% beobachtet.

\section{- Maria Weiß}

Quelle: Pressekonferenz: Aktuelle Ergebnisse aus der Nanotechnologie - Crataegus: Damit das Leben wieder rund läuft", Werder bei Potsdam (unterstützt von Dr. Willmar Schwabe $\mathrm{GmbH}$ ) 